\title{
$\mathrm{HEE} \mathrm{OH}$
}

\section{Tempered subgroups and representations with minimal decay of matrix coefficients}

\author{
Bulletin de la S. M. F., tome 126, no 3 (1998), p. 355-380 \\ <http://www.numdam.org/item?id=BSMF_1998_126_3_355_0>
}

(C) Bulletin de la S. M. F., 1998, tous droits réservés.

L'accès aux archives de la revue «Bulletin de la S. M. F. » (http: //smf.emath.fr/Publications/Bulletin/Presentation.html) implique l'accord avec les conditions générales d'utilisation (http://www.numdam.org/ conditions). Toute utilisation commerciale ou impression systématique est constitutive d'une infraction pénale. Toute copie ou impression de ce fichier doit contenir la présente mention de copyright.

\section{NumDam}


Bull. Soc. math. France,

126,1998 , p. $355-380$.

\title{
TEMPERED SUBGROUPS AND REPRESENTATIONS WITH MINIMAL DECAY OF MATRIX COEFFICIENTS
}

\author{
BY HeE OH $\left(^{*}\right)$
}

\begin{abstract}
We present a function $F$ for each simple real linear Lie group $G$ with real rank at least 2 such that $F$ bounds from above all the $K$-matrix coefficients of non-trivial irreducible spherical unitary representations of $G$ where $K$ is a maximal compact subgroup of $G$. This enables us to determine when a closed subgroup $H$ is a $(G, K)$-tempered subgroup of $G$ : for example, if the restriction $\left.F\right|_{H}$ of $F$ to $H$ lies in $L^{1-\epsilon}(H)$. We also prove that this function $F$ is the best possible for $G$ a real-split group of type $A_{n}$ or $C_{n}$ and as a consequence, we obtain that if $H$ is semisimple, then $H$ is a $(G, K)$-tempered subgroup of $G$ if and only if $\left.F\right|_{H}$ lies in $L^{1}(H)$.

RÉSUMÉ. - SOUS-GROUPES TEMPÉRÉS ET REPRÉSENTATIONS. - Nous associons une fonction $F$ à chaque groupe de Lie $G$, linéaire, réel simple de rang réel au moins 2, telle que $F$ donne une borne supérieure pour tous les coefficients matriciels $K$-finis des représentations unitaires sphériques irréductibles de $G$, où $K$ un sous-groupe compact maximal de $G$. Ceci nous permet de déterminer quand un sous-groupe fermé $H$ de $G$ est $(G, K)$-tempéré; c'est le cas par exemple si la restriction de $F$ à $H$ est dans $L^{1-\epsilon}(H)$. Nous prouvons aussi que cette fonction $F$ est la meilleure possible pour un groupe réel déployé $G$ de type $A_{n}$ ou $C_{n}$, et comme conséquence, nous obtenons que si $H$ est semi-simple, alors $H$ est un sous-groupe $(G, K)$-tempéré de $G$ si et seulement si $\left.F\right|_{H}$ est dans $L^{1}(H)$.
\end{abstract}

\section{Introduction}

Let $G$ be a connected semisimple linear Lie group and $K$ a maximal compact subgroup of $G$. We say that a unitary representation of $G$ is spherical if it has a $K$-invariant vector. For a unitary spherical represen-

(*) Texte reçu le 12 septembre 1997, révisé le 29 janvier 1998, accepté le 26 février 1998. HeE OH, Mathematics Department, Yale University, New Haven, CT 06520 (USA).

Adresse actuelle : Institute of Mathematics, The Hebrew University, Jerusalem 91904 (Israel). Email : heeoh@math.huji.ac.il.

AMS classification : 22 E 40, 22 E 46, 53 C 30, 57 S 30.

Keywords : unitary representation, tempered subgroup, compact quotient, matrix coefficient.

BULlETIN DE LA SOCIÉTÉ MATHÉMATIQUE DE FRANCE 0037-9484/1998/355/\$5.00

(C) Société mathématique de France 
tation $\rho$, we will use the term " $K$-matrix coefficients of $\rho$ " to refer to its matrix coefficients with respect to $K$-invariant unit vectors.

In this paper we are interested in the asymptotic behavior of the $K$-matrix coefficients of spherical unitary representations of $G$ when restricted to a closed subgroup $H$ of $G$. One motivation comes from the notion " $(G, K)$-tempered subgroups" of $G$ defined by Margulis [10]. That is, a closed subgroup $H$ of $G$ is called $(G, K)$-tempered if there exists a (positive) function $q \in L^{1}(H)$ such that for any non-trivial irreducible spherical unitary representation $\rho$ of $G$,

$$
|\langle\rho(h) v, w\rangle| \leq q(h)\|v\| \cdot\|w\|
$$

for all $h \in H$ and any $K$-fixed vectors $v$ and $w$. Note that any compact subgroup of $G$ is a $(G, K)$-tempered subgroup for a trivial reason. Margulis also showed in [10] that if a closed subgroup $H$ is a $(G, K)$-tempered subgroup, then for any non-compact subgroup $F$ of $H$, the quotient $G / F$ does not allow a compact quotient by a discrete subgroup of $G$ (see [6] for a survey on the general problem).

We denote by $\widehat{G}$ (resp. $\widehat{G}_{K}$ ) the set of equivalence classes of non-trivial irreducible unitary (resp. spherical) representations.

In this paper we first present a "good upper bound function" for $K$ matrix coefficients for all representations in $\widehat{G}_{K}$ for a simple real linear Lie group $G$ with real rank at least 2 . Secondly we show that in simple realsplit linear Lie group of type $A_{n}$ or $C_{n}$ this function is in fact the best possible by exhibiting a spherical representation of $G$ in $\widehat{G}_{K}$ whose $K$ matrix coefficients are bounded below by this function. We now formulate the main results.

The notation $[x]$ denotes the largest integer which is not greater than $x$.

Theorem A. - Let $G$ be a connected simple real linear Lie group with real rank $n \geq 2, K$ a maximal compact subgroup, $B$ a minimal parabolic subgroup, $A \subset B$ a maximal $\mathbb{R}$-split torus, $A^{+} \subset A$ the positive Weyl chamber given by the choice of $B$. Denote by $\Phi^{\prime}$ the set of all nonmultipliable roots in the relative root system $\Phi_{\mathbb{R}}(\mathfrak{a}, \mathfrak{g})$ where $\mathfrak{a}$ and $\mathfrak{g}$ are the Lie algebras of $A$ and $G$ respectively. Let $\alpha_{1}, \ldots, \alpha_{n}$ be the basis of $\Phi^{\prime}$ whose subscripts are determined by the highest weight given in section 2.1 .

Then for any $\epsilon>0$, there exists a constant $C$ (depending on $\epsilon$ ) such that for any $\rho \in \widehat{G}_{K}$ and $f_{0}$ a K-invariant unit vector of $\rho$,

$$
\left|\left\langle\rho(g) f_{0}, f_{0}\right\rangle\right| \leq C F(g)^{1-\epsilon} \quad \text { for any } g \in G
$$

where $F$ is the $K$-bi-invariant function defined on $A^{+}$as follows according to the type of $\Phi^{\prime}$ :

TOME $126-1998-\mathrm{N}^{\circ} 3$ 


$$
\begin{array}{ll}
\Phi^{\prime} & -\log F \\
A_{n}, n \geq 2 & \left\{\begin{array}{l}
\sum_{i=1}^{\frac{1}{2}(n-1)} \frac{1}{2} i \alpha_{i}+\sum_{i=\frac{1}{2}(n+1)}^{n} \frac{1}{2}(n-i+1) \alpha_{i} \\
\text { for } n \text { odd },
\end{array}\right. \\
\sum_{i=1}^{\frac{1}{2} n} \frac{1}{2} i \alpha_{i}+\frac{1}{4} n \alpha_{\frac{1}{2} n+1}+\sum_{i=\frac{1}{2} n+2}^{n} \frac{1}{2}(n-i+1) \alpha_{i} \\
B_{n}, n \geq 2 & \sum_{i=1}^{\left[\frac{1}{2} n\right]} i \alpha_{i}+\sum_{i=\left[\frac{1}{2} n+1\right]}^{n} \frac{1}{2} n \alpha_{i}, \\
C_{n}, n \geq 2 \text { even }, & \sum_{i=1}^{n-1} i \alpha_{i}+\frac{1}{2} n \alpha_{n}, \\
D_{n}, n \geq 4 & \sum_{i=1}^{\left[\frac{1}{2} n\right]} i \alpha_{i}+\sum_{i=\left[\frac{1}{2} n+1\right]}^{n-2} \frac{1}{2} n \alpha_{i}+\frac{1}{4} n\left(\alpha_{n-1}+\alpha_{n}\right), \\
E_{6} & \alpha_{1}+2 \alpha_{2}+2 \alpha_{3}+3 \alpha_{4}+2 \alpha_{5}+\alpha_{6}, \\
E_{7} & 2 \alpha_{1}+\frac{7}{2} \alpha_{2}+4 \alpha_{3}+6 \alpha_{4}+\frac{9}{2} \alpha_{5}+3 \alpha_{6}+\frac{3}{2} \alpha_{7}, \\
E_{8} & 2 \alpha_{1}+4 \alpha_{2}+5 \alpha_{3}+8 \alpha_{4}+7 \alpha_{5}+5 \alpha_{6}+3 \alpha_{7}+\alpha_{8}, \\
F_{4} & 2 \alpha_{1}+3 \alpha_{2}+4 \alpha_{3}+2 \alpha_{4}, \\
G_{2} & 2 \alpha_{1}+\alpha_{2} .
\end{array}
$$

Corollary B. - With the same notation as in Theorem A, let $H$ be a closed subgroup of $G$. If the restriction $\left.F\right|_{H}$ of $F$ to $H$ is in $L^{1-\epsilon}(H)$ for some $\epsilon>0$, then $H$ is a $(G, K)$-tempered subgroup of $G$.

\section{REMARKS}

1) Suppose further that $H$ is a connected semisimple Lie subgroup of $G$ such that $A \cap H$ is a maximal $\mathbb{R}$-split torus of $H$ and $B \cap H$ is a minimal parabolic subgroup of $H$. Let $\delta_{H}$ denote the modular function of $B \cap H$, that is, the product of all positive roots including the multiplicity. 
Let $\lambda_{1}, \ldots, \lambda_{m}$ be the fundamental weights of the Lie algebra of $H$ corresponding to $A \cap H$ and $B \cap H$.

For any two weights $\alpha$ and $\beta$ of the Lie algebra of $H$, we write

$$
\alpha<\beta \quad \text { if }\left(\alpha, \lambda_{j}\right)<\left(\beta, \lambda_{j}\right) \quad \text { for all } 1 \leq j \leq m
$$

Then the condition $\left.F\right|_{H} \in L^{1-\epsilon}(H)$ is equivalent to

$$
-\left.\log F\right|_{A^{+} \cap H}>\log \delta_{H}
$$

which is again equivalent to the condition $\left.F\right|_{H} \in L^{1}(H)$.

2) If the restriction $\left.F\right|_{H}$ is $L^{k-\epsilon}(H)$-integrable for some $\epsilon>0$ and some positive integer $k$, then the diagonal embedding $\delta_{k}(H)$ of $H$ into the group $\prod_{i=1}^{k} G_{i}$ is a $\left(\prod_{i=1}^{k} G_{i}, \prod_{i=1}^{k} K_{i}\right)$-tempered subgroup of $\prod_{i=1}^{k} G_{i}$ where $G_{i}=G$ and $K_{i}=K$ for all $1 \leq i \leq k$. To see this, it is enough to note that for any non-trivial irreducible spherical representation $\rho$ of $\prod_{i=1}^{k} G_{i}$, the restrictions of the $K$-matrix coefficients of $\rho$ to $\delta_{k}(H)$ are bounded by $(F \mid H)^{k(1-\epsilon)}$.

For a unitary representation $\rho$ of $G, \rho$ is said to be strongly $L^{q}$ if there is a dense subset $V$ in the Hilbert space attached to $\rho$ such that the matrix coefficients of $\rho$ with respect to the vectors in $V$ lie in $L^{q}(G)$. Let $p(G)$ be the smallest real number such that for any $\rho \in \widehat{G}, \rho$ is strongly $L^{q}$ for any $q>p(G)\left(c f\right.$. [7]). Similarly let $p_{K}(G)$ be the smallest real number such that for any $\rho \in \widehat{G}_{K}$, the $K$-matrix coefficients of $\rho$ are $L^{q}(G)$-integrable for any $q>p_{K}(G)$. The estimate of the Harish-Chandra function $\Xi$ of $G$ shows that $p_{K}(G)$ is at least $2(c f .[3])$ and hence $G$ cannot be a $(G, K)$ tempered subgroup of itself. The method used in proving Theorem A yields upper bounds for both $p(G)$ and $p_{K}(G)$.

The following follows from Remark 1 after Corollary B.

Corollary C. - With the same notation as in Theorem A, let $\delta_{G}$ be the modular function of $B$ (cf. Table 3.7). Define

$$
r(G)=\max \left\{\frac{\text { the coefficient of } \alpha_{i} \text { in } \log \delta_{G}}{\text { the coefficient of } \alpha_{i} \text { in }-\log F} ; i=1, \cdots, n\right\} .
$$

Then $p(G) \leq r(G)$ and $p_{K}(G) \leq r(G)$.

TOME $126-1998-\mathrm{N}^{\circ} 3$ 
If $G$ is split over $\mathbb{R}, r(G)$ is as follows :

\begin{tabular}{ccccccccccc}
\hline$\Phi=\Phi^{\prime}$ & $:$ & $A_{n}$ & $B_{n}$ & $C_{n}$ & $D_{n}$ & $E_{6}$ & $E_{7}$ & $E_{8}$ & $F_{4}$ & $G_{2}$ \\
$r(G)$ & $:$ & $2 n$ & $2 n$ & $2 n$ & $2(n-1)$ & 16 & 18 & 58 & 11 & 6.
\end{tabular}

For $n \geq 3$, Vogan's classification of unitary duals for $\mathrm{GL}_{n}(D)$ yields that

- for $G=\mathrm{SL}_{n}(D), D=\mathbb{R}, \mathbb{C}$ or $\mathbb{H}, p(G)$ is $2(n-1), 2(n-1)$ and $2 n-1$ respectively and

- for $\operatorname{Sp}_{2 n}(\mathbb{R})$, it follows from Howe's result in [3] that $p(G)=2 n$.

The number $p(G)$ in other classical group cases was calculated by $\mathrm{Li}[7]$ and was given an upper bound by $\mathrm{Li}$ and $\mathrm{Zhu}$ [8] in exceptional split group cases. We remark that the numbers $r(G)$ in Corollary $C$ coincide with $p(G)$ calculated in [7] for all classical real split groups except $B_{n}$ type. For a split group of type $E_{6}$, by obtaining $r(G)=16$, we improve the bound for $p(G)$ in [8].

We state a theorem which yields a necessary and sufficient condition for a closed semisimple subgroup to be a $(G, K)$-tempered subgroup in a simple real split linear Lie group $G$ of type $A_{n}$ or $C_{n}$. Let $G$ be either $\mathrm{SL}_{n}(\mathbb{R})$ or $\mathrm{Sp}_{2 n}(\mathbb{R})$. The group $\mathrm{Sp}_{2 n}(\mathbb{R})$ is defined by the bi-linear form $\left(\begin{array}{cc}0 & \bar{I}_{n} \\ -\bar{I}_{n} & 0\end{array}\right)$ where $\bar{I}_{n}$ denotes the skew diagonal $n \times n$-identity matrix. Set

$$
K=\mathrm{SO}_{n}(\mathbb{R}) \quad \text { and } \quad \operatorname{Sp}_{2 n}(\mathbb{R}) \cap \mathrm{SO}_{2 n}(\mathbb{R})
$$

respectively. Define the parabolic subgroup $P$ of $G$ as follows :

- for $G=\mathrm{SL}_{n}(\mathbb{R}), P=\left\{\left(g_{i j}\right) \in G \mid g_{i 1}=0\right.$ if $\left.i \neq 1\right\}$,

- for $G=\operatorname{Sp}_{2 n}(\mathbb{R}), P=\left\{\left(g_{i j}\right) \in G \mid g_{i 1}=0, g_{2 n j}=0\right.$ if $\left.i \neq 1, j \neq 2 n\right\}$.

Note that $P$ is the maximal parabolic subgroup which stabilizes the line $\mathbb{R} e_{1}$. We fix an ordering in the root system of $G$ so that the positive Weyl chamber $A^{+}$is as follows :

$$
\begin{array}{r}
\operatorname{SL}_{n}(\mathbb{R}), \quad A^{+}=\left\{\operatorname{diag}\left(a_{1}, \ldots, a_{n}\right) \mid \prod_{i=1}^{n} a_{i}=1,\right. \\
\left.a_{i} \geq a_{i+1} \text { for all } 1 \leq i \leq n-1\right\}, \\
\operatorname{Sp}_{2 n}(\mathbb{R}), \quad A^{+}=\left\{\operatorname{diag}\left(a_{1}, \ldots, a_{n}, a_{n}^{-1}, \ldots, a_{1}^{-1}\right) \mid a_{i} \geq a_{i+1} \geq 1\right. \\
\quad \text { for all } 1 \leq i \leq n-1\} .
\end{array}
$$

BULLETIN DE LA SOCIÉTÉ MATHÉMATIQUE DE FRANCE 
Example. - The function $F$ defined in Theorem $\mathrm{A}$ is as follows :

- for $G=\mathrm{SL}_{n}(\mathbb{R})$,

$$
F(a)= \begin{cases}\prod_{i=1}^{\frac{1}{2} n} a_{i}{ }^{-1} & \text { for } n \text { even } \\ a_{\frac{1}{2}(n+1)^{-\frac{1}{2}}} \prod_{i=1}^{\frac{1}{2}(n-1)} a_{i}^{-1} & \text { for } n \text { odd }\end{cases}
$$

- for $G=\operatorname{Sp}_{2 n}(\mathbb{R})$,

$$
F(a)=\prod_{i=1}^{n}{a_{i}}^{-1} \quad \text { where } a \in A^{+}
$$

Theorem D. - Let $G$ be $\mathrm{SL}_{n}(\mathbb{R})$ or $\mathrm{Sp}_{2 n}(\mathbb{R})$ and $P, K$ and $A^{+}$be as above.

(1) For any $\epsilon>0$, there exist constants $C_{1}$ and $C_{2}$ such that

$$
C_{1} F(a) \leq\left|\left\langle\operatorname{Ind}_{P}^{G}(I)(a) f_{0}, f_{0}\right\rangle\right| \leq C_{2} F(a)^{1-\epsilon}
$$

for any $a \in A^{+}$and for any $K$-invariant unit vector $f_{0}$ in $\operatorname{Ind}_{P}^{G}(I)$.

(2) If a closed subgroup $H$ of $G$ is $(G, K)$-tempered, $\left.F\right|_{H}$ is in $L^{1}(H)$.

(3) $A$ closed semisimple subgroup $H$ of $G$ is $(G, K)$-tempered if and only if $\left.F\right|_{H}$ is in $L^{1}(H)$.

(4) $p_{K}(G)=r(G)=p(G)$.

Acknowledgments. - I am very grateful to Professors Roger Howe and Gregory Margulis for valuable suggestions and for their encouragement. Most of this paper was written during my visit at Bielefeld University. I would like to thank Professor Abels and the Mathematics Department there for their warm hospitality. I am thankful to Professors Dave Witte, T.N. Venkatarama, Alex Eskin and the referee for many useful comments.

\section{A maximal system of strongly orthogonal roots in each irreducible root system}

2.1. - Let $\Phi$ be an irreducible reduced root system with a fixed ordering. Denote by $\Phi^{+}$the set of positive roots and by $\Delta=\left\{\alpha_{1}, \ldots, \alpha_{n}\right\}$ the set of simple roots of $\Phi$. The subscripts of $\alpha_{i}$ 's are determined by the

TOME $126-1998-\mathrm{N}^{\circ} 3$ 
following choice of the highest root [2].

\begin{tabular}{ll}
$\Phi$ & the highest root \\
\hline$A_{n}$ & $\alpha_{1}+\alpha_{2}+\cdots+\alpha_{n}$, \\
$B_{n}$ & $\alpha_{1}+2 \alpha_{2}+\cdots+2 \alpha_{n}$, \\
$C_{n}$ & $2 \alpha_{1}+2 \alpha_{2}+\cdots+2 \alpha_{n-1}+\alpha_{n}$, \\
$D_{n}$ & $\alpha_{1}+2 \alpha_{2}+\cdots+2 \alpha_{n-2}+\alpha_{n-1}+\alpha_{n}$, \\
$E_{6}$ & $\alpha_{1}+2 \alpha_{2}+2 \alpha_{3}+3 \alpha_{4}+2 \alpha_{5}+\alpha_{6}$, \\
$E_{7}$ & $2 \alpha_{1}+2 \alpha_{2}+3 \alpha_{3}+4 \alpha_{4}+3 \alpha_{5}+2 \alpha_{6}+\alpha_{7}$, \\
$E_{8}$ & $2 \alpha_{1}+3 \alpha_{2}+4 \alpha_{3}+6 \alpha_{4}+5 \alpha_{5}+4 \alpha_{6}+3 \alpha_{7}+2 \alpha_{8}$, \\
$F_{4}$ & $2 \alpha_{1}+3 \alpha_{2}+4 \alpha_{3}+2 \alpha_{4}$, \\
$G_{2}$ & $3 \alpha_{1}+2 \alpha_{2}$. \\
\hline
\end{tabular}

We define the number $N(\Phi)$ as follows :

$$
N(\Phi)= \begin{cases}{\left[\frac{1}{2}(n+1)\right]} & \text { for } \Phi=A_{n} \\ 2\left[\frac{1}{2} n\right] & \text { for } \Phi=D_{n} \\ 4 & \text { for } \Phi=E_{6} \\ \operatorname{rank}(\Phi) & \text { for } \Phi=B, C, F_{4}, G_{2}, E_{7}, E_{8}\end{cases}
$$

\subsection{Construction of some strongly orthogonal roots.}

Two roots $\alpha$ and $\beta$ are called strongly orthogonal if neither one of $\alpha \pm \beta$ is a root. Consider the family $\mathcal{S}(\Phi)$ of all subsets of $\Phi^{+}$whose elements are pairwise strongly orthogonal. We call an element $\mathcal{O}$ in $\mathcal{S}(\Phi)$ a strongly orthogonal system.

Let $f$ be the function on $\mathcal{S}(\Phi)$ given by

$$
f(\mathcal{O})=\sum_{\alpha \in \mathcal{O}} \alpha
$$

The aim of this section is to construct an element

$$
\mathcal{Q}(\Phi)=\left\{\gamma_{1}, \ldots, \gamma_{N(\Phi)}\right\}
$$

in $\mathcal{S}(\Phi)$ on which $f$ attains its maximum. For simplicity, we set $N(\Phi)=N$.

We define $\mathcal{Q}(\Phi)$ as follows :

BULLETIN DE LA SOCIÉTÉ MATHÉMATIQUE DE FRANCE 
$A_{n}\left\{\begin{array}{l}\gamma_{i}=\alpha_{i}+\cdots+\alpha_{n-i+1} \\ \gamma_{N}= \begin{cases}\alpha_{N} & \text { for } n \text { odd } \\ \alpha_{N}+\alpha_{N+1} & \text { for } n \text { even }\end{cases} \end{array}\right.$

$B_{n} \begin{cases}\gamma_{2 i-1}=\alpha_{i}+\cdots+\alpha_{n-i}+2 \alpha_{n-i+1}+\cdots+2 \alpha_{n}, \\ \gamma_{2 i}=\alpha_{i}+\cdots+\alpha_{n-i} & \text { for } i \leq\left[\frac{1}{2} n\right], \\ \gamma_{n}=\alpha_{\frac{1}{2}(n+1)}+\cdots+\alpha_{n} & \text { for } n \text { odd }\end{cases}$

$C_{n}\left\{\begin{array}{l}\gamma_{i}=2 \alpha_{i}+\cdots+2 \alpha_{n-1}+\alpha_{n} \quad \text { for } i \leq N-1, \\ \gamma_{N}=\alpha_{n}\end{array}\right.$

$D_{n}\left\{\begin{aligned} \gamma_{1}=\alpha_{1}+\cdots+\alpha_{n-2}+\alpha_{n}, \\ \gamma_{2}=\alpha_{1}+\cdots+\alpha_{n-1}, \\ \gamma_{2 i-1}=\alpha_{i}+\cdots+\alpha_{n-i}+2 \alpha_{n-i+1} \\ \quad+\cdots+2 \alpha_{n-2}+\alpha_{n-1}+\alpha_{n}, \\ \gamma_{2 i}=\alpha_{i}+\cdots+\alpha_{n-i} \quad \text { for } 3 \leq i \leq\left[\frac{1}{2} n\right]\end{aligned}\right.$

$E_{6}\left\{\begin{array}{l}\gamma_{1}=\alpha_{1}+\alpha_{2}+\alpha_{3}+2 \alpha_{4}+2 \alpha_{5}+\alpha_{6}, \\ \gamma_{2}=\alpha_{1}+\alpha_{2}+2 \alpha_{3}+2 \alpha_{4}+\alpha_{5}+\alpha_{6}, \\ \gamma_{3}=\alpha_{2}+\alpha_{3}+2 \alpha_{4}+\alpha_{5}, \\ \gamma_{4}=\alpha_{2} ;\end{array}\right.$

$E_{7}\left\{\begin{array}{l}\gamma_{1}=\alpha_{1}+\alpha_{2}+\alpha_{3}+2 \alpha_{4}+2 \alpha_{5}+\alpha_{6}+\alpha_{7} \\ \gamma_{2}=\alpha_{1}+\alpha_{2}+2 \alpha_{3}+2 \alpha_{4}+\alpha_{5}+\alpha_{6}+\alpha_{7} \\ \gamma_{3}=\alpha_{1}+\alpha_{2}+2 \alpha_{3}+2 \alpha_{4}+2 \alpha_{5}+\alpha_{6} \\ \gamma_{4}=\alpha_{1}+\alpha_{2}+\alpha_{3}+2 \alpha_{4}+\alpha_{5}+\alpha_{6} \\ \gamma_{5}=\alpha_{2}+\alpha_{3}+2 \alpha_{4}+\alpha_{5} \\ \gamma_{6}=\alpha_{2}+\alpha_{3}+2 \alpha_{4}+2 \alpha_{5}+2 \alpha_{6}+\alpha_{7} \\ \gamma_{7}=\alpha_{2}\end{array}\right.$

$E_{8}\left\{\begin{array}{l}\gamma_{1}=\alpha_{1}+2 \alpha_{2}+3 \alpha_{3}+5 \alpha_{4}+4 \alpha_{5}+3 \alpha_{6}+2 \alpha_{7}+\alpha_{8} \\ \gamma_{2}=\alpha_{1}+2 \alpha_{2}+2 \alpha_{3}+3 \alpha_{4}+3 \alpha_{5}+2 \alpha_{6}+\alpha_{7}+\alpha_{8} \\ \gamma_{3}=\alpha_{1}+2 \alpha_{2}+2 \alpha_{3}+3 \alpha_{4}+2 \alpha_{5}+2 \alpha_{6}+\alpha_{7}+\alpha_{8} \\ \gamma_{4}=\alpha_{1}+\alpha_{2}+\alpha_{3}+2 \alpha_{4}+2 \alpha_{5}+2 \alpha_{6}+2 \alpha_{7}+\alpha_{8} \\ \gamma_{5}=\alpha_{1}+\alpha_{2}+2 \alpha_{3}+2 \alpha_{4}+\alpha_{5}+\alpha_{6}+\alpha_{7}+\alpha_{8} \\ \gamma_{6}=\alpha_{1}+\alpha_{2}+2 \alpha_{3}+2 \alpha_{4}+2 \alpha_{5}+\alpha_{6}+\alpha_{7} \\ \gamma_{7}=\alpha_{1}+\alpha_{2}+\alpha_{3}+2 \alpha_{4}+\alpha_{5} \\ \gamma_{8}=\alpha_{1}+\alpha_{3}+\alpha_{4}+\alpha_{5}+\alpha_{6}\end{array}\right.$

$F_{4}\left\{\begin{array}{l}\gamma_{1}=\alpha_{1}+2 \alpha_{2}+4 \alpha_{3}+2 \alpha_{4} \\ \gamma_{2}=\alpha_{1}+2 \alpha_{2}+2 \alpha_{3}+2 \alpha_{4} \\ \gamma_{3}=\alpha_{1}+2 \alpha_{2}+2 \alpha_{3} \\ \gamma_{4}=\alpha_{1}\end{array}\right.$

TOME $126-1998-\mathrm{N}^{\circ} 3$ 
$G_{2}\left\{\begin{array}{l}\gamma_{1}=3 \alpha_{1}+2 \alpha_{2} \\ \gamma_{2}=\alpha_{1}\end{array}\right.$

The following lemma can be easily checked.

Lemma. - The set $\mathcal{Q}(\Phi)$ is a strongly orthogonal system.

Proposition 2.3. - One has $f(\mathcal{Q}(\Phi))=\max _{\mathcal{O} \in \mathcal{S}(\Phi)} f(\mathcal{O})$, that is, for any $\mathcal{O} \in \mathcal{S}(\Phi)$, the coefficient of $\alpha_{i}$ in $f(\mathcal{Q}(\Phi))$ is greater than or equal to the coefficient of $\alpha_{i}$ in $f(\mathcal{O})$ for each $1 \leq i \leq n$, where $n$ is the rank of $\Phi$.

Proof. - Let $\mathcal{O}$ be any element in $\mathcal{S}(\Phi)$. We prove this proposition by induction. We can easily check that the proposition is true for $n=2$. Suppose that $n \geq 3$.

- For $\Phi=A_{n}$, take any element in $\mathcal{O}$, say $\alpha=\alpha_{i}+\cdots+\alpha_{j-1}, i<j-1$. Then $\alpha \leq \gamma_{1}$ since $\gamma_{1}$ is the highest root. On the other hand $\mathcal{O}-\{\alpha\}$ is contained in $\left\{\alpha_{m}+\cdots+\alpha_{\ell-1} \mid m, \ell \notin\{i, j\}\right\}$, which is a root system of type $A_{n-2}$. Note that

$$
\mathcal{Q}\left(A_{n}\right) \cap\left\{\alpha_{m}+\cdots+\alpha_{\ell-1} \mid m, \ell \notin\{i, j\}\right\}=\mathcal{Q}\left(A_{n-2}\right) .
$$

Therefore by the induction assumption, $f(\mathcal{O}-\{\alpha\}) \leq f\left(\mathcal{Q}\left(A_{n-2}\right)\right)$. Hence we have $f(\mathcal{O}) \leq \gamma_{1}+f\left(\mathcal{Q}\left(A_{n-2}\right)\right) \leq f\left(\mathcal{Q}\left(A_{n}\right)\right)$, proving the claim.

- For $\Phi=B_{n}$, note that for any $\alpha \in \Phi^{+}$, we have that the coefficient of $\alpha_{1}$ in $\alpha$ is at most 1 . Write $\mathcal{O}$ as $\mathcal{O}_{1} \cup \mathcal{O}_{2}$ so that $\beta \in \mathcal{O}_{1}$ if and only if the coefficient of $\alpha_{1}$ in $\beta$ is 1 and $\mathcal{O}_{2}=\mathcal{O}_{1}^{c}$. It is not difficult to check that if three positive roots in $B_{n}$ are mutually strongly orthogonal, then the coefficient of $\alpha_{1}$ in at least one of them is 0 . Therefore $\left|\mathcal{O}_{1}\right| \leq 2$. We can easily see that for any two strongly orthogonal roots $\beta_{1}, \beta_{2} \in \Phi^{+}$such that the coefficient of $\alpha_{1}$ in $\beta_{i}$ is 1 for both $i=1,2$, we have $\beta_{1}+\beta_{2} \leq \sum_{i=1}^{n} 2 \alpha_{i}$; hence $\sum_{\beta \in \mathcal{O}_{1}} \beta \leq \gamma_{1}+\gamma_{2}$, because $\left|\mathcal{O}_{1}\right| \leq 2$ and $\gamma_{1}+\gamma_{2}=\sum_{i=1}^{n} 2 \alpha_{i}$. For $\theta \subset \Delta$, the notation $[\theta]$ denotes the set of the roots in $\Phi$ which can be expressed as integral combinations of the roots in $\theta$. Since $\mathcal{O}_{2} \subset\left[\alpha_{2}, \cdots, \alpha_{n}\right]$, $\gamma_{3}+\gamma_{4}=\sum_{i=2}^{n} 2 \alpha_{i}$ and $\left[\alpha_{2}, \cdots, \alpha_{n}\right]$ is a root system of type $B_{n-1}$, we can proceed by induction as before.

- The argument for $D_{n}$ is exactly the same as the one for $B_{n}$; so we omit it. 
- If $\Phi$ is of type $C_{n}$, write $\mathcal{O}=\mathcal{O}_{1} \cup \mathcal{O}_{2}$ so that $\beta \in \mathcal{O}_{1}$ if and only if the coefficient of $\alpha_{1}$ in $\beta$ is positive and $\mathcal{O}_{2}=\mathcal{O}_{1}{ }^{c}$. It is easy to see that $\left|\mathcal{O}_{1}\right| \leq 1$. Therefore $\sum_{\alpha \in \mathcal{O}_{1}} \alpha \leq \gamma_{1}$, for $\gamma_{1}$ is the highest root in $\Phi$. Since $\mathcal{O}_{2} \subset\left[\alpha_{2}, \cdots, \alpha_{n}\right]$, it remains to use induction process.

- For exceptional root system cases, we can prove the proposition by checking each root system case by case.

As a corollary of the above proposition, we obtain that $\mathcal{Q}(\Phi)$ is a maximal element in $\mathcal{S}(\Phi)$ with respect to the inclusion ordering.

REMARK. - I learned from E. Vinberg that this construction of a strongly orthogonal system coincides with the so called Kostant's cascade construction ( $c f .[9]$ ), if $\Phi$ is one of the types $A_{n}, C_{n}$ or $G_{2}$. But in all cases the cardinalities of the sets in Kostant's cascade construction coincide with the numbers $N(\Phi)$, which are the cardinalities of $\mathcal{Q}(\Phi)$ in our construction. We note that not all maximal strongly orthogonal systems in $\Phi$ have the same cardinality. For example, $\left\{\alpha_{2}, \alpha_{4}, 2 \alpha_{2}+3 \alpha_{2}+4 \alpha_{3}+2 \alpha_{4}\right\}$ is a maximal strongly orthogonal system in the root system of $F_{4}$.

We remark that if $\Phi$ is none of $A_{n}, C_{n}$ or $G_{2}$, the function $f$ attains its maximum in our construction but not in Kostant's cascade construction.

\section{An upper bound function for matrix coefficients in simple non-compact linear Lie groups}

3.1. - Let $G$ be a connected semisimple non-compact linear Lie group, $B$ a minimal parabolic subgroup, $A$ a maximal $\mathbb{R}$-split torus contained in $B, A^{+}$the positive Weyl chamber and $K$ a maximal compact subgroup. Consider a Cartan decomposition of $G: G=K A^{+} K$. Since the $K$-matrix coefficients of a spherical unitary representation are $K$-bi-invariant, they are determined by their restrictions to the $A^{+}$-part. Denote by $\mathfrak{g}$ the Lie algebra of $G$ and by $\mathfrak{a}$ the Lie algebra of $A$. We denote by $\Phi_{\mathbb{R}}(\mathfrak{a}, \mathfrak{g})$ the set of restricted roots of $(\mathfrak{g}, \mathfrak{a})$, which is endowed with the ordering given by $B$. If $G$ is split over $\mathbb{R}$, then $\Phi_{\mathbb{R}}(\mathfrak{a}, \mathfrak{g})$ will be simply denoted by $\Phi(\mathfrak{a}, \mathfrak{g})$. If we fix a Haar measure $\mathrm{d} g$ on $G$, then the modular function $\delta_{G}$ of $B$ is given as

$$
\delta_{G}=\prod_{\alpha \in \Phi_{\mathbb{R}}^{+}(\mathfrak{a}, \mathfrak{g})} \exp \alpha .
$$

It is well known ( $c f$. [3]) that the induced representation $\operatorname{Ind}_{B}^{G}(I)$ of the trivial representation of $B$ is irreducible and has a unique (up to a

TOME $126-1998-\mathrm{N}^{\circ} 3$ 
sign) $K$-invariant unit vector, say $f_{0}$. The matrix coefficient of $\operatorname{Ind}_{B}^{G}(I)$ defined by

$$
g \longmapsto\left\langle\operatorname{Ind}_{B}^{G}(I)(g) f_{0}, f_{0}\right\rangle
$$

is called the Harish-Chandra function of $G$, which we will denote by $\Xi_{G}$. When there is no confusion, $\Xi_{G}$ will simply be denoted by $\Xi$.

Harish-Chandra has shown the following :

Proposition $(c f .[3])$. - For any $\epsilon>0$, there exist constants $c_{1}$ and $c_{2}$ such that, for all $a \in A^{+}$

$$
c_{1} \delta_{G}^{-1 / 2}(a) \leq \Xi_{G}(a) \leq c_{2} \delta_{G}^{-1 / 2+\epsilon}(a) .
$$

Moreover the value of Harish-Chandra function $\Xi$ of $\mathrm{SL}_{2}(\mathbb{R})$ or $\mathrm{PSL}_{2}(\mathbb{R})$ at $\left(\begin{array}{cc}a_{0} & 0 \\ 0 & a_{0}^{-1}\end{array}\right)$ for $a_{0}>1$ is asymptotically $\left(\log a_{0}\right) / a_{0}$ up to some constant multiple.

3.2. - We can write the Haar measure $\mathrm{d} g$ of $G$ in terms of the Cartan decomposition $K A^{+} K$ as follows :

$$
\mathrm{d} g=\Delta(a) \mathrm{d} k_{1} \mathrm{~d} a \mathrm{~d} k_{2}
$$

where $\Delta(a)$ is a positive function on $A^{+}$satisfying

$$
d_{1}(t) \delta_{G}(a) \leq \Delta(a) \leq d_{2} \delta_{G}(a)
$$

for all $a \in\left\{a \in A^{+}|| \alpha(a) \mid \geq t\right.$ for all $\left.\alpha \in \Phi_{\mathbb{R}}^{+}(\mathfrak{a}, \mathfrak{g})\right\}$ and for some constants $d_{1}(t)$ and $d_{2}$ if $t>1$ (cf. [3]).

For a $K$-matrix coefficient $\phi(g)=\langle\rho(g) v, w\rangle$ of $\rho \in \widehat{G}_{K}$, it is well known that $\phi \in L^{p}(G)$ if and only if $\int_{A^{+}}|\phi(a)|^{p} \delta_{G}(a) \mathrm{d} a<\infty$.

Proposition 3.3. - Let $H$ be $\mathrm{SL}_{2}(\mathbb{R})$ or $\mathrm{PSL}_{2}(\mathbb{R})$. Suppose that for some $k \geq 2, H$ acts on $\mathbb{R}^{k}$ by a rational representation so that the only $H$-invariant vector is the origin. Let $H \ltimes \mathbb{R}^{k}$ be the associated semidirect product. Let $\rho$ be a unitary representation of $H \ltimes \mathbb{R}^{k}$ without any $\mathbb{R}^{k}$ invariant vectors. Then we have

$$
\left|\left\langle\left.\rho\right|_{H}(h) v, w\right\rangle\right| \leq \Xi_{H}(h)(\operatorname{dim}\langle K \cdot v\rangle \operatorname{dim}\langle K \cdot w\rangle)^{1 / 2}
$$

where $h \in H, K=S O_{2}(\mathbb{R})$ and $v$ and $w$ are $K$-finite unit vectors of $\rho$. Moreover if $\rho$ is spherical, then the $K$-matrix coefficients of $\left.\rho\right|_{H}$ are bounded by $\Xi_{H}$. 
Proof. - By [12, Thm 7.3.9], the restriction $\left.\rho\right|_{H}$ of $\rho$ to $H$ is weakly contained in the infinite sum of the regular representation of $H$. It is well known ( $c f .[4, \mathrm{Ch} . \mathrm{V}$, Thm 3.21$]$ ) that the $K$-finite (or $K$-fixed) matrix coefficients of the regular representation of $H$ satisfy the above inequality.

In the spirit of Howe's strategy (see [7]) we state the following proposition. The notation $\mathfrak{u}_{\alpha}$ for $\alpha \in \Phi_{\mathbb{R}}(\mathfrak{a}, \mathfrak{g})$ denotes the root space in $\mathfrak{g}$ corresponding to $\alpha$.

Proposition 3.4. - Let $G$ be a connected simple real split linear Lie group. Let $\left\{\beta_{1}, \ldots, \beta_{m}\right\} \subset \Phi^{+}(\mathfrak{a}, \mathfrak{g})$ be a strongly orthogonal system. Then for any $\epsilon>0$, there exists a constant $C$ such that for any $\rho \in \widehat{G}_{K}$ and $K$ fixed unit vectors $v$ and $w$ of $\rho$, and for any $a \in A^{+}$

$$
|\langle\rho(a) v, w\rangle| \leq C \prod_{i=1}^{m} \exp \left(\left(-\frac{1}{2}+\epsilon\right) \beta_{i}\right)(a) .
$$

Proof. - For each $1 \leq i \leq m$, let $H_{i}$ be the connected closed subgroup of $G$ whose Lie algebra is generated by $\mathfrak{u}_{ \pm \beta_{i}}$. Note that for each $1 \leq i \leq m$,

(1) $H_{i}$ is isomorphic to $S L_{2}(\mathbb{R})$ or $P S L_{2}(\mathbb{R})$;

(2) the subgroups $H_{i} \cap B, H_{i} \cap A$ and $H_{i} \cap K$ are a minimal parabolic subgroup, a maximal $\mathbb{R}$-split torus and a maximal compact subgroup of $H_{i}$ respectively;

(3) the positive Weyl chamber $A^{+}\left(H_{i}\right)$ of $H_{i}$ is contained in $A^{+}$.

It is not difficult to see that

$$
A^{+} \subset \prod_{i=1}^{m} A^{+}\left(H_{i}\right) C_{G}\left(\prod_{i=1}^{m} H_{i}\right)
$$

where $C_{G}\left(\prod_{i=1}^{m} H_{i}\right)$ denotes the centralizer of $\prod_{i=1}^{\substack{m \\ m}} H_{i}$. Since $\left\{\beta_{1}, \ldots, \beta_{m}\right\}$ is a strongly orthogonal system, it follows that $x_{i} x_{j}=x_{j} x_{i}$ for all $i \neq j$, $x_{i} \in H_{i}$ and $x_{j} \in H_{j}$. By looking at the root system, it is not difficult to see that for each $H_{i}$, there exists an abelian unipotent subgroup $U_{i}$ of $G$ of dimension at least 2 such that $H_{i}$ normalizes $U_{i}$ and $C_{G}\left(H_{i}\right) \cap U_{i}$ is trivial. It follows that for each $1 \leq i \leq m, H_{i} \ltimes \mathbb{R}^{k_{i}}$ can be considered to be a subgroup of $G$ where the semidirect product is as described in Proposition 3.3 and $k_{i}=\operatorname{dim} U_{i}$.

Let $\rho \in \widehat{G}_{K}$, and $v$ and $w$ be $K$-fixed unit vectors. The restriction $\rho_{\mid \Pi_{i=1}^{m} H_{i}}$ can be written as a direct integral $\int_{X} \rho_{\alpha} \mathrm{d} \mu(\alpha)$ where $X$ is some Borel measure space with measure $\mu, \alpha \in X$ and $\rho_{\alpha}$ is an irreducible

TOME $126-1998-\mathrm{N}^{\circ} 3$ 
representation of $\prod_{i=1}^{m} H_{i}$ for all $\alpha \in X$ (cf. [3]). Without loss of generality, we may assume that all $\rho_{\alpha}$ 's are non-trivial spherical representations. Any element $a \in A^{+}$can be uniquely written as $a=a_{1} \cdots a_{m} c$ where $a_{i} \in A^{+}\left(H_{i}\right)$ and $c \in C_{G}\left(\prod_{i=1}^{m} H_{i}\right)$. Write $\rho(c) v$ and $w$ as $\int v_{\alpha} \mathrm{d} \mu(\alpha)$ and $\int w_{\alpha} \mathrm{d} \mu(\alpha)$ respectively where $v_{\alpha}$ and $w_{\alpha}$ are vectors in $\rho_{\alpha}$. Since $c$ centralizes each $H_{i}, \rho(c) v$ is $K \cap H_{i}$-fixed for all $1 \leq i \leq m$. Therefore there is no loss of generality in assuming that for all $\alpha \in X, v_{\alpha}$ and $w_{\alpha}$ are $K \cap H_{i}$-fixed for all $1 \leq i \leq m$. Fix $\alpha \in X$. Then

$$
\rho_{\alpha \mid \Pi_{i=1}^{m} H_{i}}=\bigotimes_{i=1}^{m} \rho_{\alpha i}, \quad v_{\alpha}=\bigotimes_{i=1}^{m} v_{\alpha i}, \quad w_{\alpha}=\bigotimes_{i=1}^{m} w_{\alpha i},
$$

where $\rho_{\alpha i}$ is a spherical irreducible representation of $H_{i}$ and $v_{\alpha i}$ and $w_{\alpha i}$ are $K \cap H_{i}$-fixed vectors for each $1 \leq i \leq m$. By Moore's theorem ( $c f$. [12, Thm 2.1.9]), for each $1 \leq i \leq m$, the representation $\rho_{\alpha i}$ is non-trivial and $\rho_{\alpha i}$ has no $U_{i}$-invariant vector.

By Proposition 3.3, we obtain that for each $1 \leq i \leq m$,

$$
\left|\left\langle\rho_{\alpha i}\left(a_{i}\right) v_{\alpha i}, w_{\alpha i}\right\rangle\right| \leq \Xi_{H_{i}}\left\|v_{\alpha i}\right\| \cdot\left\|w_{\alpha i}\right\| .
$$

Hence

$$
\begin{aligned}
|\langle\rho(a) v, w\rangle| & \leq \int_{\alpha}\left|\left\langle\rho_{\alpha}\left(\prod_{i=1}^{m} a_{i}\right) v_{\alpha}, w_{\alpha}\right\rangle\right| \mathrm{d} \mu(\alpha) \\
& \leq \int_{\alpha} \prod_{i=1}^{m}\left|\left\langle\rho_{\alpha i}\left(a_{i}\right) v_{\alpha i}, w_{\alpha i}\right\rangle\right| \mathrm{d} \mu(\alpha) \\
& \leq \int_{\alpha} \prod_{i=1}^{m}\left(\Xi_{H_{i}}\left(a_{i}\right)\left\|v_{\alpha i}\right\| \cdot\left\|w_{\alpha i}\right\|\right) \mathrm{d} \mu(\alpha) \\
& =\prod_{i=1}^{m} \Xi_{H_{i}}\left(a_{i}\right)\|\rho(c) v\| \cdot\|w\| \\
& \leq \prod_{i=1}^{m} \Xi_{H_{i}}\left(a_{i}\right)\|v\| \cdot\|w\|=\prod_{i=1}^{m} \Xi_{H_{i}}\left(a_{i}\right) .
\end{aligned}
$$

Note that the modular function $\delta_{H_{i}}$ of $H_{i} \cap B$ is equal to $\exp \left(\beta_{i}\right)$. Hence by Proposition 3.1, for each $i$, there exists a constant $C_{i}$ (not depending on $a$ ) such that

$$
\Xi_{H_{i}}\left(a_{i}\right) \leq C_{i} \exp \left(-\frac{1}{2}+\epsilon\right) \beta_{i}(a) .
$$

This proves the proposition. 
3.5. Proof of Theorem A. - It is well known [1, Thm 7.2] that $G$ contains a connected simple closed subgroup $G_{0}$ such that $G_{0}$ is split over $\mathbb{R}$, rank $G_{0}=\mathbb{R}$-rank $G$ and $\Phi^{\prime}$ is isomorphic to $\Phi\left(\mathfrak{g}_{0}, \mathfrak{g}_{0} \cap \mathfrak{a}\right)$ where $\Phi^{\prime}$ is the set of all non-multipliable roots in $\Phi_{\mathbb{R}}(\mathfrak{g}, \mathfrak{a})$ and $\mathfrak{g}_{0}$ is the Lie algebra of $G_{0}$. Recall the strongly orthogonal system $\mathcal{Q}\left(\Phi^{\prime}\right)=\left\{\gamma_{1}, \ldots, \gamma_{N\left(\Phi^{\prime}\right)}\right\}$ of $\Phi^{\prime}$ we constructed in section 2.2.

The notation $\mathfrak{u}_{\alpha}$ for $\alpha \in \Phi^{\prime}$ denotes the one-dimensional root subalgebra of $\mathfrak{g}_{0}$. Set $N=N\left(\Phi^{\prime}\right)$. For each $1 \leq i \leq N$, we define $H_{i}$ to be the connected closed subgroup of $G_{0}$ whose Lie algebra is generated by $\mathfrak{u}_{ \pm \gamma_{i}}$.

- Case $\Phi^{\prime} \neq D_{n=2 k+1}$. - We note that the restriction $F \mid A^{+}$of the function $F$ to $A^{+}$in Theorem $A$ is equal to $\prod_{i=1}^{N} \delta_{H_{i}}{ }^{-1 / 2}$ or equivalently,

$$
F \mid A^{+}=\prod_{i=1}^{N} \exp \left(-\frac{1}{2} \gamma_{i}\right)
$$

Then Theorem $A$ follows from Proposition 3.4.

- Case $\Phi^{\prime}=D_{n=2 k+1}$. - In this case, we define $H_{N}^{\prime}$ to be the connected closed subgroup of $G_{0}$ whose Lie algebra is generated by $\mathfrak{u}_{ \pm\left(\alpha_{k+1}+2\left(\alpha_{k+2}+\cdots+\alpha_{n-2}\right)+\alpha_{n-1}+\alpha_{n}\right)}, \mathfrak{u}_{ \pm \alpha_{k}}$ and $\mathfrak{u}_{ \pm \alpha_{k+1}}$. Note that the Lie algebra of $H_{N}^{\prime}$ is isomorphic to that of $\mathrm{SO}(3,3)$. We have that

$$
\delta_{H_{N}^{\prime}}=\exp \left(4 \alpha_{k}\right)\left(\prod_{i=k+1}^{n-2} \exp \left(6 \alpha_{i}\right)\right) \exp \left(3 \alpha_{n-1}\right) \exp \left(3 \alpha_{n}\right)
$$

By [7, Lemma 4.1], the restriction $\rho \mid H_{N}^{\prime}$ is strongly $L^{4+\epsilon}$ for any $\rho \in \widehat{G}$. This implies [3, Cor. 7.2] that the restrictions to $H_{N}^{\prime}$ of the $K$-finite matrix coefficients (with respect to unit vectors) of $\rho$ are bounded by $\Xi_{H_{N}^{\prime}}^{1 / 2}$. It is not difficult to see from the proof of Proposition 3.4 that, when we replace $H_{N}$ by $H_{N}{ }^{\prime}$, a statement similar to Proposition 3.4 holds, that is, the $K$-matrix coefficients of $\rho$ for any $\rho \in \widehat{G}_{K}$ are bounded above by $\left(\prod_{i=1}^{N-1} \delta_{H_{i}}^{-1 / 2}\right) \delta_{H_{N}^{\prime}}^{-1 / 4}$. Therefore it remains to observe that the function $F$ in Theorem $A$ is given by

$$
\begin{aligned}
F \mid A^{+}=\left(\prod_{i=1}^{n-2} \exp \left(-\frac{1}{2} \gamma_{i}\right)\right) \exp \left(\alpha_{k}\right)( & \left.\prod_{i=k+1}^{n-2} \exp \left(\frac{3}{2} \alpha_{i}\right)\right) \\
& \times \exp \left(\frac{3}{4} \alpha_{n-1}\right) \exp \left(\frac{3}{4} \alpha_{n}\right)
\end{aligned}
$$

TOME $126-1998-\mathrm{N}^{\circ} 3$ 
which is equal to $\left(\prod_{i=1}^{n-2} \delta_{H_{i}}^{-1 / 2}\right) \delta_{H_{N}^{\prime}}^{-1 / 4}$, to complete the proof.

REMARK. - The results in section 2.2 show that the function $F$ is the best possible upper bound for $K$-matrix coefficients, which can be obtained using Proposition 3.4 when $\Phi^{\prime} \neq D_{n=2 k+1}$. Note that when $\Phi^{\prime}=D_{n=2 k+1}$, we improved $F$ by replacing one $\mathrm{SL}_{2}(\mathbb{R})$ by $\operatorname{SO}(3,3)$.

Corollary 3.6. - With $G, \Phi^{\prime}$ and $\alpha_{1}, \cdots, \alpha_{n}$ as in section 3.5, suppose that $\mathcal{O}=\left\{\beta_{1}, \ldots, \beta_{t}\right\}$ is a strongly orthogonal system of $\Phi^{\prime}$ and that for some number $r$, the coefficient of $\alpha_{j}$ in $\sum_{i=1}^{t} r \beta_{i}$ is strictly bigger than the coefficient of $\alpha_{j}$ in $2 \log \left(\delta_{G}\right)$ for each $1 \leq j \leq n$. Then

$$
p(G) \leq r \quad \text { and } \quad p_{K}(G) \leq r .
$$

3.7. - In each simple real-split Lie group $G$, the modular function $\delta_{G}$ of $B$ is given as below ( $c f .[2]$ ), from which the remark after Corollary $\mathrm{C}$ follows.

$\Phi \quad \log \delta$

$$
\begin{array}{ll}
A_{n} & \sum_{i=1}^{n} i(n-i+1) \alpha_{i}, \\
B_{n} & \left(\sum_{i=1}^{n-1}\left(2 n i-i^{2}\right) \alpha_{i}\right)+n^{2} \alpha_{n}, \\
& \left(\sum_{i=1}^{n}\left(2 n i-i^{2}+i\right) \alpha_{i}\right)+\frac{1}{2} n(n+1) \alpha_{n}, \\
& \left(\sum_{i=1}^{n-2}\left(2 n i-i^{2}-i\right) \alpha_{i}\right)+\frac{1}{2} n(n-1)\left(\alpha_{n-1}+\alpha_{n}\right), \\
D_{n} & 16 \alpha_{1}+22 \alpha_{2}+30 \alpha_{3}+42 \alpha_{4}+30 \alpha_{5}+16 \alpha_{6}, \\
E_{6} & 34 \alpha_{1}+49 \alpha_{2}+66 \alpha_{3}+96 \alpha_{4}+75 \alpha_{5}+52 \alpha_{6}+27 \alpha_{7}, \\
E_{7} & 92 \alpha_{1}+136 \alpha_{2}+182 \alpha_{3}+270 \alpha_{4}+220 \alpha_{5}+168 \alpha_{6}, \\
E_{8} & 16 \alpha_{1}+30 \alpha_{2}+42 \alpha_{3}+22 \alpha_{4}, \\
F_{4} & 10 \alpha_{1}+6 \alpha_{2} .
\end{array}
$$




\section{Spherical unitary representations with minimal decay in $\mathrm{SL}_{n}(\mathbb{R})$ and $\operatorname{Sp}_{2 n}(\mathbb{R})$}

4.1. - In this section we will show that the upper bound function $F$ we obtained in Theorem $\mathrm{A}$ is the best possible when $G=\operatorname{SL}_{n}(\mathbb{R})$ or $\operatorname{Sp}_{2 n}(\mathbb{R})$. This will be proved by showing that there exists a spherical unitary representation of $G$ whose $K$-matrix coefficients are bounded from below by a constant multiple of $F$. Those representations are the induced representations $\operatorname{Ind}_{P}^{G}(I)$ of the trivial representation where $P$ is the maximal parabolic subgroup which stabilizes the line $\mathbb{R} e_{1}$.

4.2. - For the rest of section 4 , let $G$ be either $\mathrm{SL}_{n}(\mathbb{R})$ or $\operatorname{Sp}_{2 n}(\mathbb{R})$. The group $\operatorname{Sp}_{2 n}(\mathbb{R})$ is defined by the bi-linear form $\left(\begin{array}{cc}0 & \bar{I}_{n} \\ -\bar{I}_{n} & 0\end{array}\right)$ where $\bar{I}_{n}$ denotes the skew diagonal $(n \times n)$-identity matrix. Set

$$
K=\mathrm{SO}_{n}(\mathbb{R}) \quad \text { and } \quad \mathrm{Sp}_{2 n}(\mathbb{R}) \cap \mathrm{SO}_{2 n}(\mathbb{R})
$$

respectively. Define the maximal parabolic subgroup $P$ of $G$ as follows :

- for $G=\mathrm{SL}_{n}(\mathbb{R})$,

$$
P=\left\{\left(g_{i j}\right) \in G \mid g_{i 1}=0 \text { if } i \neq 1\right\}
$$

- for $G=\operatorname{Sp}_{2 n}(\mathbb{R})$,

$$
P=\left\{\left(g_{i j}\right) \in G \mid g_{i 1}=0, g_{(2 n) j}=0 \text { if } i \neq 1, j \neq 2 n\right\} .
$$

We fix an ordering in the root system of $G$ so that the positive Weyl chamber $A^{+}$is as follows :

$$
\begin{gathered}
\mathrm{SL}_{n}(\mathbb{R}), \quad A^{+}=\left\{\operatorname{diag}\left(a_{1}, \cdots, a_{n}\right) \mid \prod_{i=1}^{n} a_{i}=1,\right. \\
\left.a_{i} \geq a_{i+1} \text { for all } 1 \leq i \leq n-1\right\} ; \\
\mathrm{Sp}_{2 n}(\mathbb{R}), \quad A^{+}=\left\{\operatorname{diag}\left(a_{1}, \cdots, a_{n}, a_{n}^{-1}, \cdots, a_{1}^{-1}\right) \mid a_{i} \geq a_{i+1} \geq 1\right. \\
\quad \text { for all } 1 \leq i \leq n-1\} .
\end{gathered}
$$

4.3. - We recall the formula for the matrix coefficients of the induced representation $\operatorname{Ind}_{P}^{G}(I)$ ( $c f$. [5]). Consider the Langlands decomposition of $P: P=M A_{P} N$. Denote by $\bar{N}$ the unipotent radical of the opposite parabolic subgroup to $P$ with the common Levi subgroup $M A_{P}$.

If $g$ decomposes under the decomposition $G=K M A_{P} N$, we denote by $\exp H(g)$ the $A_{P}$-component of $g$. It is well known that the representation

TOME $126-1998-\mathrm{N}^{\circ} 3$ 
space of $\operatorname{Ind}_{P}^{G}(I)$ of the trivial representation $I$ of $P$ can be realized as $L^{2}(\bar{N}, d x)$. If $g$ decomposes under $\bar{N} M A_{P} N$ as

$$
g=\bar{n}(g) m(g) a(g) n(g),
$$

then the action is given by

$$
\operatorname{Ind}_{P}^{G}(I)(g) f(x)=e^{-\delta_{0}\left(\log a\left(g^{-1} x\right)\right)} f\left(\bar{n}\left(g^{-1} x\right)\right)
$$

for any $f \in L^{2}(\bar{N}, d x)$ and $x \in \bar{N}$, where $\delta_{0}$ is the half sum of positive $N$-roots.

Define the vector $f_{0}$ of $\operatorname{Ind}_{P}^{G}(I)$ as follows :

$$
f_{0}(x)=\mathrm{e}^{-\delta_{0}(H(x))} .
$$

It is not difficult to see that $f_{0}$ is $K$-fixed and the matrix coefficient of $\operatorname{Ind}_{P}^{G}(I)$ with respect to $f_{0}$ is as follows :

$$
\left\langle\operatorname{Ind}_{P}^{G}(I)(g) f_{0}, f_{0}\right\rangle=\int_{\bar{N}} \mathrm{e}^{-\delta_{0}\left(\log a\left(g^{-1} x\right)\right)} \mathrm{e}^{-\delta_{0}\left(H\left(\bar{n}\left(g^{-1} x\right)\right)\right)} \mathrm{e}^{-\delta_{0}(H(x))} \mathrm{d} x .
$$

4.4. - Theorem $D$ follows from the following proposition and Theorem A.

Proposition. - There exists a constant $C$ such that

$$
C F(a) \leq\left|\left\langle\operatorname{Ind}_{P}^{G}(I)(a) f_{0}, f_{0}\right\rangle\right|
$$

where $a \in A^{+}$and $F$ is as in Theorem $A$

Proof of Proposition 4.4.

- Case $G=\mathrm{SL}_{n}(\mathbb{R}), n \geq 3$.

Denote by $\tilde{a}$ the matrix $\operatorname{diag}\left(a_{1}, \cdots, a_{n}\right) \in \mathrm{SL}_{n}(\mathbb{R})$ and by $x$ the matrix in $\bar{N}$ whose first column is $\left(1, x_{2}, \cdots, x_{n}\right)$, that is, $x . e_{1}=\left(1, x_{2}, \ldots, x_{n}\right)$. To simplify notation, set $x_{1}=1$.

The decomposition of $\tilde{a}^{-1} x$ under $\bar{N} M A_{P} N$ is as follows :

$$
\begin{gathered}
a\left(\tilde{a}^{-1} x\right)=\operatorname{diag}\left(a_{1}, a_{1}^{-1 /(n-1)}, \cdots, a_{1}^{-1 /(n-1)}\right), \\
\bar{n}\left(\tilde{a}^{-1} x\right) \cdot e_{1}=\left(1, \frac{a_{1}}{a_{2}} x_{2}, \ldots, \frac{a_{1}}{a_{n}} x_{n}\right) .
\end{gathered}
$$

Then $\exp \delta_{0}\left(a\left(\tilde{a}^{-1} x\right)\right)=a_{1}^{-n / 2}$ and $H(x)=\left\|x \cdot e_{1}\right\|=\sqrt{\sum_{i=1}^{n} x_{i}{ }^{2}}$. 
Therefore

$$
\begin{aligned}
\left\langle\operatorname{Ind}_{P}^{G}(I)(\tilde{a}) f_{0}, f_{0}\right\rangle & =\int_{\bar{N}} a_{1}{ }^{n / 2}\left\|\bar{n}\left(\tilde{a}^{-1} x\right) . e_{1}\right\|^{-n / 2}\left\|x . e_{1}\right\|^{-n / 2} \mathrm{~d} x \\
& =\int_{\mathbb{R}^{n-1}}\left(\sum_{i=1}^{n}\left(\frac{1}{a_{i}}\right)^{2} x_{i}^{2}\right)^{-n / 4}\left(\sum_{i=1}^{n} x_{i}^{2}\right)^{-n / 4} \mathrm{~d} m
\end{aligned}
$$

where $\mathrm{d} m$ is the standard measure in $\mathbb{R}^{n-1}$.

Set $k=\left[\frac{1}{2}(n+1)\right]$ and let $T$ be the following set :

$$
\begin{aligned}
\left\{\left(x_{2}, \cdots, x_{n}\right) \mid 0 \leq x_{i} \leq 1 \text { for } 2 \leq i \leq k-1,1 \leq x_{k} \leq 2,\right. & \\
& \left.x_{i} \leq \frac{a_{i}}{a_{k}} x_{k} \text { for } k+1 \leq i \leq n\right\} .
\end{aligned}
$$

Note that if $\left(x_{2}, \ldots, x_{n}\right) \in T$, then for each $1 \leq i \leq n$, we have

$$
x_{i} \leq 2 \quad \text { and } \quad \frac{x_{i}}{a_{i}} \leq \frac{x_{k}}{a_{k}} .
$$

Thus for $\left(x_{2}, \cdots, x_{n}\right) \in T$, we have

$$
\left(\sum_{i=1}^{n}\left(\frac{1}{a_{i}}\right)^{2} x_{i}^{2}\right)^{-n / 4}\left(\sum_{i=1}^{n} x_{i}^{2}\right)^{-n / 4} \geq C a_{k}{ }^{n / 2}
$$

for some constant $C>0$. Therefore

$$
\left|\left\langle\operatorname{Ind}_{P}^{G}(I)(\tilde{a}) f_{0}, f_{0}\right\rangle\right| \geq C \int_{T} a_{k}^{n / 2} \mathrm{~d} m \geq C a_{k}{ }^{n / 2} \prod_{i=k+1}^{n}\left(\frac{a_{i}}{a_{k}}\right) \geq C F(\tilde{a}) .
$$

- Case $G=\operatorname{Sp}_{2 n}(\mathbb{R}), n \geq 2$.

For $\tilde{a}=\operatorname{diag}\left(a_{1}, \cdots, a_{n}, a_{n}^{-1}, \cdots, a_{1}^{-1}\right) \in \operatorname{Sp}_{2 n}(\mathbb{R})$, we have

$$
\begin{aligned}
\left\langle\operatorname{Ind}_{P}^{G}(I)(\tilde{a}) f_{0}, f_{0}\right\rangle=\int_{\mathbb{R}^{2 n-1}}\left(\sum_{i=1}^{n}\left(\frac{x_{i}}{a_{i}}\right)^{2}\right. & \left.+\sum_{i=1}^{n}\left(a_{i} y_{i}\right)^{2}\right)^{-n / 2} \\
& \times\left(\sum_{i=1}^{n} x_{i}^{2}+\sum_{i=1}^{n} y_{i}^{2}\right)^{-n / 2} \mathrm{~d} m
\end{aligned}
$$

where $x_{1}=1$. Let $T$ be the following set :

$$
\begin{aligned}
\left\{\left(x_{1}, \cdots, x_{n}, y_{n}, \cdots, y_{1}\right) \mid 1 \leq y_{n}\right. & \leq 2, y_{i} \leq \frac{a_{n}}{a_{i}} y_{n} \\
0 & \left.\leq x_{i} \leq 1,0 \leq y_{i} \leq 2 \text { for } 1 \leq i \leq n\right\} .
\end{aligned}
$$

TOME $126-1998-\mathrm{N}^{\circ} 3$ 
Note that if $\left(x_{1}, \ldots, x_{n}, y_{n}, \ldots, y_{1}\right) \in T$, then

$$
x_{i} \leq a_{i} a_{n} y_{n}
$$

since $a_{i} \geq 1$ for all $1 \leq i \leq n$. Therefore

$$
\left|\left\langle\operatorname{Ind}_{P}^{G}(I)(\tilde{a}) f_{0}, f_{0}\right\rangle\right| \geq C \int_{T}\left(a_{n} y_{n}\right)^{-n} \mathrm{~d} m \geq C F(\tilde{a})
$$

where $C$ is some positive constant, finishing the proof.

\section{5. $(G, K)$-tempered subgroups and finite dimensional representations}

5.1. - Let $H$ be a linear connected non-compact semisimple Lie group. Let $B_{H}$ be a minimal parabolic subgroup, $A_{H}$ a maximal $\mathbb{R}$-split torus contained in $B_{H}$ and $K_{H}$ a maximal compact subgroup of $H$. Consider a Cartan decomposition of $H: H=K_{H} A_{H}^{+} K_{H}$. Let $A$ be the torus of $\mathrm{SL}_{n}(\mathbb{R})$ consisting of all the diagonal elements and $A^{+}$the positive Weyl chamber of $\mathrm{SL}_{n}(\mathbb{R})$ given by

$$
A^{+}=\left\{\operatorname{diag}\left(a_{1}, \ldots, a_{n}\right) \mid a_{i} \geq a_{i+1} \text { for all } 1 \leq i \leq n-1\right\} .
$$

Let $\pi$ be a representation of $H$ to $\mathrm{SL}_{n}(\mathbb{R})$ such that $\pi\left(A_{H}\right) \subset A$. For each $1 \leq i \leq n$, we define a weight $\beta_{i}$ of $d \pi$ by

$$
\beta_{i}(X)=(i, i) \text {-entry of the matrix } \mathrm{d} \pi(X) \text { for } X \in \log A_{H},
$$

where $\mathrm{d} \pi$ denotes the differential of $\pi$. Denote by $W$ the Weyl group of $\mathrm{SL}_{n}(\mathbb{R})$. Using the well known isomorphism of $W$ with the symmetric group on $n$ letters, we can consider the action of $W$ on $\left\{\beta_{1}, \ldots, \beta_{n}\right\}$ by $w\left(\beta_{i}\right)=\beta_{w(i)}$ for each $1 \leq i \leq n$.

For each $w \in W$, set

$$
\mathfrak{a}_{w}=\left\{X \in \log \left(A_{H}^{+}\right) \mid w\left(\beta_{i}\right)(X) \geq w\left(\beta_{i+1}\right)(X) \text { for all } 1 \leq i \leq n-1\right\} .
$$

Note that since

$$
\mathfrak{a}_{w}=\left\{X \in \log \left(A_{H}^{+}\right) \mid \mathrm{d} \pi(X) \in w^{-1}\left(\log A^{+}\right) w\right\},
$$

we have that $\log \left(A_{H}^{+}\right)=\bigcup_{w \in W} \mathfrak{a}_{w}$. It is not difficult to see that we can choose a subset $W_{0} \subset W$ (not unique) so that $\log \left(A_{H}^{+}\right)=\bigcup_{w \in W_{0}} \mathfrak{a}_{w}$, the interior of $\mathfrak{a}_{w}$ is non-empty for each $w \in W_{0}$, and the interiors of $\mathfrak{a}_{w}$ 's, $w \in W_{0}$ are disjoint. For example, if $\pi\left(A_{H}^{+}\right) \subset A^{+}$, then we can choose $W_{0}$ to consist of only the identity element of $W$.

We keep the above notation, such as $H, A^{+}, \pi, \beta_{1}, \ldots, \beta_{n}, W_{0}, \mathfrak{a}_{w}$, etc., for the rest of Chapter 5 . Recall also that $\delta_{H}$ denotes the modular function of $B_{H}$. 
The following is an application of Theorem $D$ when $G=\mathrm{SL}_{n}(\mathbb{R})$.

Corollary. - The subgroup $\pi(H)$ is an $\left(S L_{n}(\mathbb{R}), S O_{n}(\mathbb{R})\right)$-tempered subgroup if and only if the following holds : for each $w \in W_{0}$ and all $X \in \mathfrak{a}_{w}$

- if $n$ is even

$$
w\left(\beta_{1}\right)(X)+\cdots+w\left(\beta_{n / 2}\right)(X)>\log \left(\delta_{H}\right)(X) ;
$$

- if $n$ is odd

$$
w\left(\beta_{1}\right)(X)+\cdots+w\left(\beta_{(n-1) / 2}\right)(X)+\frac{1}{2} w\left(\beta_{(n+1) / 2}\right)(X)>\log \left(\delta_{H}\right)(X) .
$$

Proof. - Note that

$$
\int_{A_{H}^{+}}(F \circ \pi) \delta_{H} \mathrm{~d} a=\sum_{w \in W_{0}} \int_{\exp \mathfrak{a}_{w}}(F \circ \pi) \delta_{H} \mathrm{~d} a .
$$

On the other hand, on each $\mathfrak{a}_{w}$, the restriction of $-\log F \circ \pi$ to $\log A_{H}^{+}$ is equal to the function in the left in the above inequality (see Example before Theorem $\mathrm{D}$ ). This proves the claim by Theorem $D$.

ExAmPle. - If $H$ is simple and Ad is the adjoint representation of $H$, we can consider $\operatorname{Ad}(H)$ to be a subgroup of $\mathrm{SL}_{n}(\mathbb{R})$ where $n=$ $\operatorname{dim}(\operatorname{Lie}(H))$. Since the restriction of $-\log F \circ$ Ad to $\log A_{H}^{+}$is equal to $\log \delta_{H}$, we have that $\operatorname{Ad}(H)$ is not an $\left(\mathrm{SL}_{n}(\mathbb{R}), \mathrm{SO}_{n}(\mathbb{R})\right)$-tempered subgroup by the above corollary.

5.2. - Let $\lambda_{1}, \ldots, \lambda_{k}$ the fundamental weights of the Lie algebra of $H$ corresponding to $A_{H}^{+}$. For any weights $\gamma_{1}$ and $\gamma_{2}$ of the Lie algebra of $H$, we define a partial order $>$ so that $\gamma_{1}>\gamma_{2}$ if and only if $\left(\gamma_{1}, \lambda_{j}\right)>\left(\gamma_{2}, \lambda_{j}\right)$ for all $1 \leq j \leq k$. This is equivalent to saying that the coefficient of each simple root in $\gamma_{1}-\gamma_{2}$ is positive, or $\gamma_{1}(X)>\gamma_{2}(X)$ for all $X \in \log A_{H}^{+}$.

If $\lambda$ is the highest weight of an irreducible representation, then the lowest weight, which we will denote by $\Lambda(\lambda)$, is given by

$$
\left(\Lambda(\lambda), \lambda_{j}\right)=-\left(\lambda, i\left(\lambda_{j}\right)\right) \text { for each } 1 \leq j \leq k
$$

where $i$ is the opposition involution of the root system of $\operatorname{Lie}(H)(c f .[11])$.

$$
\text { TOME } 126-1998-\mathrm{N}^{\circ} 3
$$


Corollary. - Let $H$ be a linear connected semisimple Lie group and $\pi$ an irreducible representation with the highest weight $\lambda$. Suppose that

$$
\lambda-\Lambda(\lambda)>2 \log \delta_{H} .
$$

Then $\pi(H)$ is an $\left(\mathrm{SL}_{n}(\mathbb{R}), \mathrm{SO}_{n}(\mathbb{R})\right)$-tempered subgroup.

Proof. - Let $w \in W_{0}$. Since $\lambda$ and $\Lambda(\lambda)$ are the highest weight and the lowest weight of $\pi$ respectively, it follows from the definition of $\mathfrak{a}_{w}$ that

$$
w\left(\beta_{1}\right)=\lambda \text { and } w\left(\beta_{n}\right)=\Lambda(\lambda) .
$$

Let $X$ be any element in $\mathfrak{a}_{w}$. Since $w\left(\beta_{i}\right)(X) \geq w\left(\beta_{i+1}\right)(X)$ for each $1 \leq i \leq n-1$, we have that if $n$ is even,

$$
2 \sum_{i=1}^{n / 2} w\left(\beta_{i}\right)(X) \geq 2 w\left(\beta_{1}\right)(X)+\sum_{i=2}^{n-1} w\left(\beta_{i}\right)(X)
$$

and if $n$ is odd

$$
\sum_{i=1}^{(n-1) / 2} w\left(\beta_{i}\right)(X)+w\left(\beta_{(n+1) / 2}\right)(X) \geq 2 w\left(\beta_{1}\right)(X)+\sum_{i=2}^{n-1} w\left(\beta_{i}\right)(X) .
$$

On the other hand, since $\sum_{i=1}^{n} \beta_{i}=0$,

$$
2 w\left(\beta_{1}\right)+\sum_{i=2}^{n-1} w\left(\beta_{i}\right)=w\left(\beta_{1}\right)-w\left(\beta_{n}\right)
$$

which is equal to $\lambda-\Lambda(\lambda)$. Therefore the assumption that $\lambda-\Lambda(\lambda)>$ $2 \log \delta_{H}$ implies the inequalities in Corollary 5.1, finishing the proof.

Remark. - By the remark prior to Corollary 5.2 and the fact that

$$
\left(\log \delta_{H}, \lambda_{j}\right)=\left(\log \delta_{H}, i\left(\lambda_{j}\right)\right) \text { for each } 1 \leq j \leq k,
$$

we have that if $\lambda>\log \delta_{H}$, then $\lambda-\Lambda(\lambda)>2 \log \delta_{H}$; so the hypothesis of the above corollary is satisfied.

EXAmPLE. - If $H=\mathrm{SL}_{k+1}(\mathbb{R})$ in Corollary 5.2, then

$$
\lambda-\Lambda(\lambda)>2 \log \delta_{H}
$$

BULLETIN DE LA SOCIÉTÉ MATHÉMATIQUE DE FRANCE 
is equivalent to the following :

$$
c_{j}+c_{k+1-j}>2 j(k+1-j) \text { for } 1 \leq j \leq k
$$

where $c_{j}=\left(\lambda, \lambda_{j}\right)$.

ExAmple 5.3. - The following examples are applications of Corollary 5.1 .

1) If $\pi$ is an irreducible representation of $\mathrm{SL}_{2}(\mathbb{R})$ into $\mathrm{SL}_{n}(\mathbb{R})$, then it is well known that $\left(\lambda, \lambda_{1}\right)=\frac{1}{2}(n-1)$; whereas $\left(\log \delta_{H}, \lambda_{1}\right)=1$. Therefore $\pi\left(\mathrm{SL}_{2}(\mathbb{R})\right)$ is an $\left(\mathrm{SL}_{n}(\mathbb{R}), \mathrm{SO}_{n}(\mathbb{R})\right)$-tempered subgroup if and only if $n \geq 4$.

2) The embedding of $\mathrm{SL}_{k}(\mathbb{R})$ as the first $k$ by $k$ diagonal block matrix in $\mathrm{SL}_{n}(\mathbb{R})$ is not an $\left(\mathrm{SL}_{n}(\mathbb{R}), \mathrm{SO}_{n}(\mathbb{R})\right)$-tempered subgroup for any positive integers $k$ and $n$.

3) For matrices $A$ of order $m$ and $B$ of order $k$, the Kronecker product $A \otimes B$ of $A$ and $B$ is the matrix of order $m k$ such that the $(i j)$-matrix block of $A \otimes B$ is $a_{i j} B$ where $a_{i j}$ is the $(i j)$-entry of $A$.

The group $\mathrm{SL}_{m}(\mathbb{R}) \otimes I_{k}$ is an $\left(\mathrm{SL}_{m k}(\mathbb{R}), \mathrm{SO}_{m k}(\mathbb{R})\right)$-tempered subgroup if and only if $k>2(m-1)$.

5.4. - In this section we consider the case when $\pi$ is symplectic or orthogonal. It is worthwhile to state the following fact, which enables us to tell when an irreducible representation $\pi$ with the highest weight $\lambda$ has such a property.

Theorem ( $c f .[11$, Ch. 3, Thm 2.15]). - The representation $\pi$ is selfdual if and only if $\lambda=-\Lambda(\lambda)$. In such cases, $\pi$ is orthogonal (resp. symplectic) if $\sum_{j=1}^{k}\left(\log \delta_{H}, \lambda_{j}\right)\left(\lambda, \lambda_{j}\right)$ is even (resp. odd).

We remark that all finite dimensional irreducible representations of $H$ are self-dual unless $H$ is of type $A_{n}, D_{2 k+1}$ or $E_{6}$.

5.5. - We have the following corollary of Theorem $\mathrm{D}$ when $G=$ $\mathrm{Sp}_{n}(\mathbb{R})$, which is analogous to Corollaries 5.1 and 5.2.

We use the same realization of $\operatorname{Sp}_{n}(\mathbb{R})$ as in section 4.2 so that a positive Weyl chamber of $\operatorname{Sp}_{n}(\mathbb{R})$ is the following :

$$
\begin{array}{r}
\operatorname{Sp}_{n}(\mathbb{R}) \cap A^{+}=\left\{\operatorname{diag}\left(a_{1}, \cdots, a_{n / 2}, a_{n / 2}^{-1}, \cdots, a_{1}^{-1}\right) \mid\right. \\
\left.a_{i} \geq a_{i+1} \geq 1 \text { for all } 1 \leq i \leq \frac{1}{2} n-1\right\} .
\end{array}
$$

TOME $126-1998-\mathrm{N}^{\circ} 3$ 
COROllary. - Let $H$ be a linear connected semisimple Lie group and $\pi$ a representation such that $\pi(H) \subset S p_{n}(\mathbb{R})$.

(1) The subgroup $\pi(H)$ is an $\left(\operatorname{Sp}_{n}(\mathbb{R}), \mathrm{Sp}_{n}(\mathbb{R}) \cap \mathrm{SO}_{n}(\mathbb{R})\right)$-tempered subgroup if and only if for each $w \in W_{0}$,

$$
w\left(\beta_{1}\right)(X)+\cdots+w\left(\beta_{n / 2}\right)(X)>\log \delta_{H}(X) \text { for all } X \in \mathfrak{a}_{w} .
$$

(2) Furthermore assume that $\pi$ is irreducible with the highest weight $\lambda$. Suppose that

$$
\lambda>\log \delta_{H}
$$

Then $\pi(H)$ is an $\left(\operatorname{Sp}_{n}(\mathbb{R}), \operatorname{Sp}_{n}(\mathbb{R}) \cap \mathrm{SO}_{n}(\mathbb{R})\right)$-tempered subgroup.

Proof. - The proof of the first claim is similar to that of Corollary 5.1; so we will omit it. Since $\lambda$ is the highest weight, $w\left(\beta_{1}\right)=\lambda$ for each $w \in W_{0}$. Since $w\left(\beta_{i}\right)(X) \geq 0$ for any $X \in \mathfrak{a}_{w}$ and each $1 \leq i \leq \frac{1}{2} n$, we have $\sum_{i=1}^{n / 2} w\left(\beta_{i}\right)(X) \geq \lambda(X)$. Now the second claim follows from the first one.

5.6. - We consider a realization of $\mathrm{SO}(m, n-m), m=\left[\frac{1}{2} n\right]$ so that a positive Weyl chamber of $\mathrm{SO}(m, n-m)$ is given by $\mathrm{SO}(m, n-m) \cap A^{+}$, that is, if $n$ is even,

$$
\left\{\operatorname{diag}\left(a_{1}, \cdots, a_{m}, a_{m}^{-1}, \cdots, a_{1}^{-1}\right) \mid a_{i} \geq a_{i+1} \geq 1 \text { for all } 1 \leq i \leq m-1\right\}
$$

and if $n$ is odd,

$$
\left\{\operatorname{diag}\left(a_{1}, \cdots, a_{m}, 1, a_{m}^{-1}, \cdots, a_{1}^{-1}\right) \mid a_{i} \geq a_{i+1} \geq 1 \text { for all } 1 \leq i \leq m-1\right\}
$$

Corollary. - Let $H$ be a linear connected semisimple Lie group and $\pi$ an $n$-dimensional irreducible representation with the highest weight $\lambda$ such that $\pi(H) \subset \mathrm{SO}(m, n-m)$ where $m=\left[\frac{1}{2} n\right]$. Suppose that

$$
\lambda>\log \delta_{H}
$$

Then $\pi(H)$ is an $\left(\mathrm{SO}(m, n-m), S O(m, n-m) \cap \mathrm{SO}_{n}(\mathbb{R})\right)$-tempered subgroup.

Proof. - Consider the case when $n$ is even. Let $p=\left[\frac{1}{4} n\right]$. Then for any $w \in W_{0}$ and any $X \in \mathfrak{a}_{w}$, the function $F$ in Theorem $\mathrm{A}$ is such that

$$
-\log F \circ \pi(X)=w\left(\beta_{1}\right)(X)+\cdots+w\left(\beta_{p}\right)(X) .
$$


Therefore by the same argument as in the previous corollary, it is enough to show that

$$
w\left(\beta_{1}\right)(X)+\cdots+w\left(\beta_{p}\right)(X)>\log \delta_{H}(X) .
$$

This is true since $w\left(\beta_{i}\right)(X) \geq 0$ for all $1 \leq i \leq p$ and $w\left(\beta_{1}\right)=\lambda$. The proof in the case when $n$ is odd is similar.

ExAMPLE. - If $H=\mathrm{SL}_{k+1}(\mathbb{R})$ and $c_{j}=\left(\lambda, \lambda_{j}\right)$ for $1 \leq j \leq k$, then $\pi$ is self-dual if and only if $c_{j}=c_{k+1-j}$ for $1 \leq j \leq k$, and the condition $\lambda>$ $\log \delta_{H}$ is equivalent to the condition $c_{j}>2 j(k+1-j)$ for each $j=1, \cdots, k$. Therefore with these two conditions satisfied, if $\sum_{i=1}^{k} i(k+1-i) c_{i}$ is even, then $\pi\left(\mathrm{SL}_{k+1}(\mathbb{R})\right)$ is an $\left(\mathrm{SO}(m, n-m), \mathrm{SO}(m, n-m) \cap \mathrm{SO}_{n}(\mathbb{R})\right)$-tempered subgroup where $m=\left[\frac{1}{2} n\right]$, and if $\sum_{i=1}^{k} i(k+1-i) c_{i}$ is odd, then $\pi(H)$ is an $\left(\operatorname{Sp}_{n}(\mathbb{R}), \operatorname{Sp}_{n}(\mathbb{R}) \cap \mathrm{SO}_{n}(\mathbb{R})\right)$-tempered subgroup.

Moreover in the case when $H=\mathrm{SL}_{2}(\mathbb{R})$ and $\pi$ is an $n$-dimensional irreducible representation with $n \geq 4$ ( $c f$. Example 5.3), the subgroup $\pi\left(\mathrm{SL}_{2}(\mathbb{R})\right)$ is $\left(\operatorname{Sp}_{n}(\mathbb{R}), \mathrm{Sp}_{n}(\mathbb{R}) \cap \mathrm{SO}_{n}(\mathbb{R})\right)$-tempered if $n$ is even; otherwise it is $\left(\mathrm{SO}(m, n-m), S O(m, n-m) \cap \mathrm{SO}_{n}(\mathbb{R})\right)$-tempered.

5.7. Unipotent tempered subgroups. - Lastly we give examples of some unipotent tempered subgroups of $G=\mathrm{SL}_{n}(\mathbb{R})$. In order to apply Theorem $\mathrm{D}$ when $H$ is not semisimple, we need to know how each element of $H$ decomposes under the Cartan decomposition of $G$.

Consider the decomposition of the element $v_{s}=\left(\begin{array}{ll}1 & s \\ 0 & 1\end{array}\right)$ as $k_{1} a k_{2}$ under the Cartan decomposition of $\mathrm{SL}_{2}(\mathbb{R})$ with $K=\mathrm{SO}_{2}(\mathbb{R})$ and $A$ the torus consisting of all the diagonal elements. Since $v_{s}\left(v_{s}\right)^{t}=k_{1} a^{2} k_{1}^{-1}$, the eigenvalues of $a^{2}$ coincide with those of $v_{s}\left(v_{s}\right)^{t}$. If $a=\operatorname{diag}\left(b, b^{-1}\right)$, then

$$
b=\sqrt{\frac{1}{2}\left(2+s^{2}+s \sqrt{s^{2}+4}\right)} .
$$

Consider the one parameter unipotent subgroup $U_{i j}$ of $\mathrm{SL}_{n}(\mathbb{R})$ consisting of the elements $u_{i j}(s)=I+s E_{i j}, s \in \mathbb{R}$, where $i \neq j$ and $E_{i j}$ is the elementary matrix whose non-zero entry is 1 only at $(i, j)$. We keep the same notation as in section 5.1. Then the $A^{+}$-component of $u_{i j}(s)$ under the Cartan decomposition of $\mathrm{SL}_{n}(\mathbb{R})$ is $\operatorname{diag}\left(b, 1, \cdots, 1, b^{-1}\right)$ where

$$
b=\sqrt{\frac{1}{2}\left(2+s^{2}+s \sqrt{s^{2}+4}\right)}
$$

by the previous argument.

Therefore $F\left(u_{i j}(s)\right)$ is equal to $\left(\sqrt{\frac{1}{2}\left(2+s^{2}+s \sqrt{s^{2}+4}\right)}\right)^{-1}$.

TOME $126-1998-\mathrm{N}^{\circ} 3$ 
Proposition. - Let $n \geq 2$ and $i \neq j$.

(1) For any $\epsilon>0$, the restriction $\left.F\right|_{U_{i j}}$ is $L^{1+\epsilon}\left(U_{i j}\right)$-integrable; hence $U_{i j}$ is not an $\left(\mathrm{SL}_{n}(\mathbb{R}), \mathrm{SO}_{n}(\mathbb{R})\right)$-tempered subgroup.

(2) The diagonal embedding

$$
\delta\left(U_{i j}\right)=\left\{(g, g) \in \mathrm{SL}_{n}(\mathbb{R}) \times \mathrm{SL}_{n}(\mathbb{R}) \mid g \in U_{i j}\right\}
$$

is an $\left(\mathrm{SL}_{n}(\mathbb{R}) \times \mathrm{SL}_{n}(\mathbb{R}), \mathrm{SO}_{n}(\mathbb{R}) \times \mathrm{SO}_{n}(\mathbb{R})\right)$-tempered subgroup.

Proof. - The part (1) is clear. For the second claim, see the remark following Corollary B.

Now consider the unipotent one-parameter subgroup $U$ of $\mathrm{SL}_{4}(\mathbb{R})$ consisting of the elements

$$
U(s)=\left(\begin{array}{cccc}
1 & s & 0 & 0 \\
0 & 1 & 0 & 0 \\
0 & 0 & 1 & s \\
0 & 0 & 0 & 1
\end{array}\right), \quad s \in \mathbb{R} .
$$

It is easy to see that the following proposition holds.

Proposition 5.8. - The subgroup $U$ is an $\left(\mathrm{SL}_{4}(\mathbb{R}), \mathrm{SO}_{4}(\mathbb{R})\right)$-tempered subgroup.

\section{BIBLIOGRAPHIE}

[1] Borel (A.), Tits (J.). - Groupes réductifs, Publ. Math. IHES, 27, 1965 , p. $55-151$.

[2] Bourbaki (N.). - Groupes et Algèbres de Lie, Chap. IV-VI. Hermann, Paris, 1968.

[3] Howe (R.E.). - On a notion of rank for unitary representations of the classical groups, Harmonic analysis and group representations II Circlo, Palazzone della Scuola normale superiore, CIME, 1980, p. 223-331.

[4] Howe (R.E.), Tan (E.C.). - Non-Abelian Harmonic Analysis. Springer-Verlag, 1992. 
[5] Knapp (A.W.). - Representation theory of Semisimple groups - An overview based on examples. - Princeton University Press, 1986.

[6] Kobayashi (T.). - Discontinuous groups and Clifford-Klein forms of pseudo-Riemannian homogeneous manifolds, in Lecture Notes of the European School on Group theory, Perspectives in Math., Algebraic and Analytic methods in Representation theory, ed. B. Ørsted and H. Schlichtkrull, t. 17, 1996, p. 99-165.

[7] Li (J-S.). - The minimal decay of matrix coefficients for classical groups, Math. Appl. (Harmonic Analysis in China), t. 327, 1996, p. $146-169$.

[8] Li (J-S.), ZHU (C-B.). - On the decay of matrix coefficients for exceptional groups, Math. Ann., t. 305, 1996, p. 249-270.

[9] Lipsman (R.L.), Wolf (J.A.). - Canonical semi-invariants and the Plancherel formula for parabolic groups, Trans. AMS, t. 269, 1982, p. 111-131.

[10] Margulis (G.A.). - Existence of compact quotients of homogeneous spaces, measurably proper actions and decay of matrix coefficients, Bull. Soc. Math. France, t. 125, 1997, p. 447-456.

[11] Onishchik (A.), Vinberg (E.). - Lie groups and Lie algebras III. Springer-Verlag, 1993 .

[12] Zimmer (R.). - Ergodic theory and semisimple groups. - Birkhäuser, Boston, 1985 .

TOME $126-1998-\mathrm{N}^{\circ} 3$ 